\title{
ISSN 0825-0305 \\ Computation of spectral gap for a colored disordered lattice gas
}

\author{
Bey Touati Ali, Halim Zeghdoudi and Hacène Boutabia \\ Laboratory of Applied Mathematics, Mohamed Khider University of Biskra, 07000, Algeria
}

Received 09 August 2011; Accepted 24 September 2011

Copyright (c) 2011, Journal Afrika Statistika. All rights reserved

\begin{abstract}
We consider a system of colored disordered lattice gas in a volume $\Lambda$ of $\mathbb{Z}^{d}$ which plays an important role in the study of hydrodynamic limit. A new computation method for the canonical measures for such a system has been established in [10]. We use those results and base on them computation method for the related spectral gap which in turn has important application in hydrodynamic.

Résumé. On considère un système de particules multicolorées, disposées dans un volume fini $\Lambda \subset \mathbb{Z}^{d}$, muni d'un désordre. On se propose de calculer pour ce système le trou spectral du générateur associé, le trou spectral jouant un rôle important dans l'étude de la limite hydrodynamique. Une méthode de calcul des mesures canoniques pour un tel système a été établie dans [10]. Les résultats précédents nous ont permis de donner une forme explicite au trou spectral qui, son tour possède des applications importantes dans l'étude de la limite hydrodynamique.
\end{abstract}

Key words: Markov Generator; Spectral Gap; Exclusion Processes.

AMS 2010 Mathematics Subject Classification : 60K35, 60F10, 60J25.

\section{Introduction}

As in Faggionnato and Martellini [7] we consider an interacting particle system related to conduction of free electrons in doped crystals that can be described as follows : a particle sitting on a site $x$ of the cubic lattice $Z^{d}$ waits an exponential time and then attempts to jump to a neighbor site $y$. If the site $y$ is occupied then the jump is canceled, otherwise it is achieved with a rate $c_{x y}^{\alpha}$ depending only on the values $\left(\alpha_{x}, \alpha_{y}\right)$ of some external quenched disorder field $\left\{\alpha_{x}\right\}_{x \in \mathbb{Z}^{d}}$ that, for simplicity, we assume to be a

Bey Touati Ali: touatibey@yahoo.fr

Halim Zeghdoudi: hzeghdoudi@yahoo.fr

Hacène Boutabia: boutabiah@yahoo.fr 
collection of i.i.d. bounded random variables. Given an external chemical potential $\lambda$, the Hamiltonian of the system is defined as $H(\eta)=-\sum_{x}\left(\alpha_{x}+\lambda\right) \eta_{x}$, where $\eta_{x}$ is the particle occupation number at site $x$. Several investigations are been undertaken to determine the speed of convergence to equilibrium of conservative stochastic dynamics. To attack this question it is necessary to estimate the spectral gap of the corresponding Markov generator or to calculate it. In this direction an important achievement is the diffusive estimates established for Kawasaki dynamics in high temperature by Lu and Yau [9] and by Cancrini and Martinelli [2]. Recently, Boudou et al. [1] developed a general technique based on a Bochner-type identity, to estimate spectral gap of class of Markov generator. According Caputo [3] their strategy can be generalized to a colored disordered lattice gas.

Results of Dermoune and Heinrich [4] were a first step to get the hydrodynamic limit of this colored disordered simple exclusion process similar to the one described in Faggionnato and Martinelli [7]. The aim of this paper is to add another step to this hydrodynamic limit. More precisely, we compute the spectral gap for colored disordered lattice gas of exclusion processes which plays an important role in the study of hydrodynamic. We also remark that the spectral gap does not depend on disorder $\alpha$.

The paper is organized as follows. In Section 2, we introduce the preliminaries and the notations and deal with dynamics in a volume $\Lambda$, and introduce the notions of Markov generators, grand canonicals, canonical measures and spectral gaps. Section 3 is devoted to state the main results and its proofs.

\section{Preliminaries and notations}

Consider a finite subset $\Lambda$ of the d-dimensional lattice $Z^{d}$. To each site $x$, we assign a disorder, that is a random variable $\alpha_{x}$. By a particle configuration we mean an application $\eta=\left\{\eta_{x}, x \in \Lambda\right\} \in\{-1,0,+1\}^{\Lambda}$ such that:

$$
\eta_{x}=\left\{\begin{array}{l}
+1 \text { if there is a blue particle at } x \\
0 \quad \text { if there is no particle at } x \\
-1 \text { if there is a white particle at } x
\end{array}\right.
$$

As in [7], we assume that the $\alpha_{x}$ 's are i.i.d, and bounded by some constant $B$. The corresponding product measure (resp. expectation) on $\Omega_{D}=[-B, B]^{\Lambda}$ will be denoted by $P($ resp. $E)$. We set for simplicity

$$
\eta_{x}^{+}=1_{\left\{\eta_{x}=1\right\}}, \eta_{x}^{-}=1_{\left\{\eta_{x}=-1\right\}}
$$

so that

$$
\eta_{x}=\eta_{x}^{+}-\eta_{x}^{-} \text {and }\left|\eta_{x}\right|=\eta_{x}^{+}+\eta_{x}^{-}
$$

where $\eta_{x}^{+}, \eta_{x}^{-}$are Bernoulli variables satisfying $\eta_{x}^{+} \eta_{x}^{-}=0$.

Dynamics in the volume $\Lambda$. Consider a (particles) configuration $\left\{\eta_{x}, x \in \Lambda\right\}$ which is simply denoted by $\eta$. If $\{x, y\}$ is a pair of sites, we denoted by $\eta^{x, y}$ the configuration derived from $\eta$ by permuting $\eta_{x}$ with $\eta_{y}$. Namely, $\eta_{x}^{x, y}=\eta_{y}, \eta_{y}^{x, y}=\eta_{x}$ and the rest is

Journal home page: www.jafristat.net 
unchanged. The dynamics of the particles is given by a Markov process $\left\{\eta(t), t \in \mathbb{R}^{+}\right\}$ and then can be described as follows : a particle at $x$ waits an exponential time and attempts to jump to a neighbor site $y$. If this site is occupied then the jump is aborted, otherwise it is realized with a probabilistic rate

$$
c_{x, y}^{\alpha}(\eta)=f_{e}\left(\alpha_{x},\left|\eta_{x}\right|, \alpha_{y},\left|\eta_{y}\right|\right)
$$

where $f_{e}$ is a bounded function on $(R \times\{0,1\})^{2}$ satisfying [7].

Markov generator. These conditions allow us to define a disordered Markov generator $L$. Disordered means depending on the random collection $\alpha=\left\{\alpha_{x}, x \in \Lambda\right\}$ which is also called disorder. The mentioned generator $L:=L_{\Lambda}^{\alpha}$ is given for a bounded function $f$ on $\{-1,0,1\}^{\Lambda}$ by:

$$
\mathbf{L} f(\eta)=\sum_{x, y \in \Lambda} c_{x, y}^{\alpha}(\eta)\left[f\left(\eta^{x, y}\right)-f(\eta)\right]
$$

Grand canonical and canonical measures. Given real numbers $\lambda^{+}, \lambda^{-}$. We set $\lambda=$ $\ln \left(e^{\lambda^{+}}+e^{\lambda^{-}}\right)$, and we consider the product Gibbs measure $\mu=\mu^{\alpha, \lambda^{+}, \lambda^{-}}$on $\{-1,0,1\}^{\Lambda}$ defined by

$$
\mu\left(\eta_{x}^{\varepsilon}\right)=\frac{\exp \left(\alpha_{x}+\lambda^{\varepsilon}\right)}{1+\exp \left(\alpha_{x}+\lambda\right)}(\text { for } x \in \Lambda \text { and } \epsilon \in\{-,+\})
$$

Thus for all $\eta \in\{-1,0,1\}^{\Lambda}$ we have

$$
\mu(\{\eta\})=Z^{-1} \exp (-H(\eta)),
$$

where $Z$ is a normalizing constant and $H$ is the Hamiltonian defined by:

$$
H(\eta)=-\sum_{x \in \Lambda}\left[\alpha_{x}\left|\eta_{x}\right|+\lambda^{+} \eta_{x}^{+}+\lambda^{-} \eta_{x}^{-}\right] .
$$

The probability measure $\mu$ is called the grand canonical measure on $\Lambda$ with disorder configuration $\alpha$ and chemical potential couple $\left(\lambda^{+}, \lambda^{-}\right)$. Besides, in order to count blue and white particles in $\Lambda$, we introduce for $\epsilon \in\{-,+\}$ the number of $\epsilon$-particles that is :

$$
N_{\Lambda}^{\epsilon}(\eta)=\sum_{x \in \Lambda} \eta_{x}^{\epsilon}
$$

Consider the random variables $m_{\Lambda}^{+}=\frac{N_{\Lambda}^{+}}{|\Lambda|}$ and $m_{\Lambda}^{-}=\frac{N_{\Lambda}^{-}}{|\Lambda|}(|\Lambda|$ denotes the cardinality of the set $\Lambda$ ), which are the densities of blue and white particles.

Let $\left(m^{+}, m^{-}\right) \in\{0,1 /|\Lambda|, 2 /|\Lambda|, \ldots ., 1\}^{2}$ be a couple such that $m=m^{+}+m^{-} \leq 1$. From [2], we know that there exists a unique real $\lambda$ called empirical chemical potential and written $\lambda_{\Lambda}(\alpha, m)$. The grand canonical Gibbs probability measure $\mu_{\Lambda}^{\alpha, \lambda}$ on $\{0,1\}^{\Lambda}$ satisfies (cf. [7])

$$
\mu_{\Lambda}^{\alpha, \lambda}\left(m_{\Lambda}^{+}+m_{\Lambda}^{-}\right)=m .
$$

Journal home page: www.jafristat.net 
The relation between $\mu$ and $\mu_{\Lambda}^{\alpha, \lambda}$ is :

$$
\mu(f \circ|.|)=\mu_{\Lambda}^{\alpha, \lambda}(f),
$$

for all bounded function $f$ on $\{0,1\}^{\Lambda}$. We also have the corresponding canonical measure :

$$
\nu(.)=\mu(. \mid S), \text { where } S=\left\{N_{\Lambda}^{+}=N^{+}, N_{\Lambda}^{-}=N^{-}\right\},
$$

which in turn does not depend on $\left(\lambda^{+}, \lambda^{-}\right)$. It was shown in [7] that for a such couple $\left(m^{+}, m^{-}\right)$and for almost all $\alpha$, there exists a unique couple $\left(\lambda^{+}, \lambda^{-}\right)$depending on $\left(\Lambda, m^{+}, m^{-}, \alpha\right)$ such that

$$
\mu\left(N_{\Lambda}^{\epsilon}\right)=N^{\epsilon},(\forall \epsilon \in\{-,+\})
$$

Spectral gap. Set the Dirichlet form defined by

$$
D(f)=\frac{1}{|\Lambda|} \sum_{x, y \in \Lambda} \nu\left(\left|f\left(\eta^{x, y}\right)-f(\eta)\right|^{2}\right)
$$

and

$$
\mathcal{P} f=\frac{1}{|\Lambda|} \sum_{y \in \Lambda} \nu\left(f \mid \eta_{y}\right),\left(\text { for every } f \in L^{2}(\nu)\right) .
$$

Note that $I-P$ is a non-negative, bounded self-adjoint operator on $L^{2}(\nu)$. Any constant is an eigenfunction with eigenvalue 0 and the spectral gap $\lambda=\lambda(|\Lambda|)$ is defined as

$$
\lambda(|\Lambda|)=\inf _{f \in L^{2}(\nu), \nu(f)=0} \frac{\nu(f(I-\mathcal{P}) f)}{\nu\left(f^{2}\right)},
$$

where $\nu(f)$ stands for the expectation $\int f d \nu$. Note that this linear operator $P$ on $L^{2}(\nu)$ preserves positivity, and is of norm less or equal to one, and satisfies $P I=I$. These properties ensure that $P-I$ is a Markov generator. Moreover, note that $P-I$ has reversible (and thus invariant) measure $\nu$ since $\nu(f(P-I) g)=\nu(g(P-I) f$ for all $f$, $g \in L^{\dot{2}}(\nu)$.

As well, we assume that the random variables $\eta \mapsto \eta_{x}, x \in \Lambda$ are independent with respect to $\mu$ (w.r.t $\mu$ ) and we will use the notation

$$
\xi_{x}(\eta)=\mathbf{1}_{[\eta(x) \neq 0]},
$$

for $x \in \Lambda$ so that $\xi(\eta) \in\{0,1\}^{\Lambda}$ denotes the configuration of occupied sites associated to $\eta$.

Motivation. As said previously, results of [4], [5] and [6] set first steps to get the hydrodynamic limit of this colored disordered simple exclusion process as introduced in [7]. We are going to compute in this paper its spectral gap as defined above.

In this way, Let $s_{1}<s_{2}<\ldots<s_{2|\Lambda|}$ be eigenvalues of $2|\Lambda|$ by $2|\Lambda|$ blocks symmetric matrix $X$, so that $s_{1}=s_{1}(|\Lambda|)$ is the smallest eigenvalue of matrix $A=\left[\begin{array}{cc}\mathbf{Y} & \mathbf{Z} \\ \mathbf{Z} & \mathbf{Y}\end{array}\right]$, where 
$Y=\left(y_{i j}\right)$ is a $|\Lambda|$ by $|\Lambda|$ matrix with $y_{i i}=1+\frac{1}{|\Lambda|(|\Lambda|-1)}, y_{i j}=\frac{1}{|\Lambda|(|\Lambda|-1)}, \forall i \neq j$ and $Z=\left(z_{i j}\right)$ is a $|\Lambda|$ by $|\Lambda|$ matrix with $z_{i i}=\frac{|\Lambda|-1}{|\Lambda|}, z_{i j}=0, \forall i \neq j$.

Remark 1. In multicolored case (tree colors), the matrix $A$ is given by

$$
A=\left[\begin{array}{lll}
Y & Z & Z \\
Z & Y & Z \\
Z & Z & Y
\end{array}\right]
$$

\section{The main result}

Our main results of this paper are the following theorems. First recall the results of [10] in :

Theorem 1. For all $\epsilon, \epsilon^{\prime} \in\{-,+\}$ and $x, y \in \Lambda$, we have for $|\Lambda| \geq 3$,

and

$$
\begin{gathered}
\nu\left(\eta_{x}^{\epsilon} \eta_{y}^{\epsilon^{\prime}}\right)=\frac{N^{\epsilon} N^{\epsilon^{\prime}}}{|\Lambda|(|\Lambda|-1)} e^{\alpha_{x}\left(1-\xi_{x}(\eta)\right)+\alpha_{y}\left(1-\xi_{y}(\eta)\right)} \\
\nu\left(\eta_{x}^{\epsilon} \eta_{y}^{\epsilon}\right)=\frac{N^{\epsilon}\left(N^{\epsilon}-1\right)}{|\Lambda|(|\Lambda|-1)} e^{\alpha_{x}\left(1-\xi_{x}(\eta)\right)+\alpha_{y}\left(1-\xi_{y}(\eta)\right)}
\end{gathered}
$$

$$
\nu\left(\eta_{x}^{\epsilon}\right)=\frac{N^{\epsilon}}{|\Lambda|} e^{\alpha_{x}\left(1-\xi_{x}(\eta)\right)}
$$

Here is our main result.

Theorem 2. For $f \in L^{2}(\nu)$ with $\nu(f)=0$ and $|\Lambda| \geq 3$. The spectral gap is equal to

$$
\lambda(|\Lambda|)=\frac{1}{|\Lambda|}
$$

\section{Proof of Theorem 2.}

Step 1. As shown in [3], it is sufficient to prove Theorem 2 for $f=\sum_{x \in \Lambda} f_{x}^{-} \overline{\eta_{x}^{-}}+f_{x}^{+} \overline{\eta_{x}^{+}}$, where $\overline{\eta_{x}^{\epsilon}}=\eta_{x}^{\epsilon}-\nu\left(\eta_{x}^{\epsilon}\right)$, with the row $\left(f_{x}^{\epsilon}, x \in \Lambda, \epsilon=\{-,+\}\right)$ are real numbers.

Now, we take the matrix representations $\nu\left(f^{2}\right)$ and $\nu(f P f)$ used in [6]. Let for $x, y \in \Lambda$ and $\epsilon, \epsilon^{\prime} \in\{-,+\}$,

$$
\left(C_{x y}^{\epsilon \epsilon^{\prime}}\right)=\nu\left(\eta_{x}^{\epsilon} ; \eta_{y}^{\epsilon^{\prime}}\right), R_{x y}^{\epsilon \epsilon^{\prime}}=\frac{C_{x y}^{\epsilon \epsilon^{\prime}}}{\sqrt{C_{x x}^{\epsilon \epsilon^{\prime} C_{y y}^{\epsilon \epsilon^{\prime}}}}} .
$$

$C=\left(C_{x y}^{\epsilon \epsilon^{\prime}}\right)$ is a $2|\Lambda|$ by $2|\Lambda|$ covariance matrix and $R=\left(R_{x y}^{\epsilon \epsilon^{\prime}}\right)$ is the corresponding correlation matrix. Let $\left(f_{x}^{\epsilon}, x \in \Lambda, \epsilon=-,+\right) \in R^{2|\Lambda|}$. We will identify $f=\sum_{x \in \Lambda} f_{x}^{-} \overline{\eta_{x}}+$ $f_{x}^{+} \overline{\eta_{x}^{+}}$.

Journal home page: www.jafristat.net 
Hence we can write

$$
\nu\left(f^{2}\right)=f C f^{T}
$$

and

$$
\nu(f \mathcal{P} f)=\frac{1}{|\Lambda|} f C D C f^{T}
$$

where $D=\left(D_{x y}^{\epsilon \epsilon^{\prime}}\right)$ is the $2|\Lambda|$ by $2|\Lambda|$ symmetric matrix defined by

$$
D_{x x}^{\epsilon \epsilon^{\prime}}=\frac{\left(2 \delta_{\epsilon}^{\epsilon^{\prime}}-1\right) C_{x x}^{\epsilon \epsilon^{\prime}}}{\operatorname{det}\left(C_{x x}\right)}, D_{x y}^{\epsilon \epsilon^{\prime}}=0 \text { for } x \neq y .
$$

Now, we shall find a non negative matrix $Q$ and a row $g$ such that

$$
\nu\left(f^{2}\right)=g Q g^{T}, \nu(f \mathcal{P} f)=\frac{1}{|\Lambda|} g Q^{2} g^{T} .
$$

Now if we set

$$
\begin{gathered}
h=Q^{1 / 2} g \text { and } \Gamma=I-Q, \\
\nu\left(f^{2}\right)=h h^{T}
\end{gathered}
$$

and

$$
\nu(f(I-\mathcal{P}) f)=h\left(\frac{(|\Lambda|-1) I}{|\Lambda|}+\frac{\Gamma}{|\Lambda|}\right) h^{T},
$$

we get by Theorem 1

$$
\inf _{f \in L^{2}(\nu), \nu(f)=0} \frac{\nu(f(I-\mathcal{P}) f)}{\nu\left(f^{2}\right)}=\inf _{h \neq 0} \frac{h \mathbf{A} h^{T}}{h h^{T}} .
$$

There exists an orthogonal matrix $P$ such that $A=P^{-1} S P$ where $S=$ $\operatorname{Diag}\left(s_{1}, s_{2}, \ldots, s_{2|\Lambda|}\right)$ is a diagonal matrix whose diagonal elements are eigenvalues of $A$. If $P$ is partitioned as $P=\left(e_{1}, e_{2}, \ldots, e_{2|\Lambda|}\right)$ where $e_{i}$ is an eigenvector of $A$, then $A$ can be written as

$$
\mathbf{A}=\sum_{i=1}^{2|\Lambda|} e_{i} e_{i}^{T}
$$

If $h \neq 0$ we have

$$
h=\sum_{i=1}^{2|\Lambda|} h_{i} e_{i}
$$

We derive from this that

$$
\frac{h \mathbf{A} h^{T}}{h h^{T}}=\frac{\sum_{i=1}^{2|\Lambda|} s_{i} h_{i}^{2}}{\sum_{i=1}^{2|\Lambda|} h_{i}^{2}} \geq s_{1} \frac{\sum_{i=1}^{2|\Lambda|} h_{i}^{2}}{\sum_{i=1}^{2|\Lambda|} h_{i}^{2}}=s_{1}
$$

Journal home page: www.jafristat.net 
Now, if we choose $h=e_{1}$, we have

$$
\inf _{h \neq 0} \frac{h \mathbf{A} h^{T}}{h h^{T}}=s_{1}
$$

Finally by combining (6) and (7) we prove the first step.

\section{Step 2.}

Now, we seek the value exact of the smallest eigenvalue of matrix $A$. Let $A=\left[\begin{array}{ccc}Y & Z & Z \\ Z & Y & Z \\ Z & Z & Y\end{array}\right]$, for simplicity we put $|\Lambda|=m, \alpha=\frac{1}{m(m-1)}$ and $\beta=\frac{m-1}{m}$. Hence $Y$ is a $m \times m$ matrix with entries $y_{i i}=1+\alpha, y_{i j}=\alpha$ if $i \neq j$ and $Z$ is a $m \times m$ matrix with entries $z_{i i}=\beta, z_{i j}=0$ if $i \neq j$.

Let

$$
\mathcal{P}(\lambda)=\operatorname{det}\left(\mathbf{A}-\lambda I_{3 m}\right)=\left|\begin{array}{ccc}
Y-\lambda I_{m} & Z & Z \\
Z & Y-\lambda I_{m} & Z \\
Z & Z & Y-\lambda I_{m}
\end{array}\right|,
$$

where $I_{3 m}$ (resp. $I_{m}$ ) denotes the identity matrix of size $3 m \times 3 m$ (resp. $m \times m$ ), be the characteristic polynomial of $A$.

According to [8] and the fact that $Y-\lambda I_{m}$ and $Z$ commute, $P(\lambda)$ can be rewritten as

$$
P(\lambda)=\operatorname{det}\left(\left(Y-\lambda I_{m}-Z\right)\left(\left(Y-\lambda I_{m}\right)^{2}+Z\left(Y-\lambda I_{m}\right)-2 Z^{2}\right)\right) .
$$

Since

$$
\left(Y-\lambda I_{m}\right)^{2}=\left(Y-\lambda I_{m}-Z\right)\left(Y-\lambda I_{m}+2 Z\right),
$$

we get

$$
\mathbf{P}(\lambda)=\left(\operatorname{det}\left(Y-\lambda I_{m}-Z\right)\right)^{2} \operatorname{det}\left(Y-\lambda I_{m}+2 Z\right) .
$$

Now we want to compute $\operatorname{det}\left(Y-\lambda I_{m}-Z\right)=P_{m}$. Recall that

$$
P_{m}=\left|\begin{array}{ccccc}
1+\alpha-\beta-\lambda & \alpha & \alpha & \cdots & \alpha \\
\alpha & 1+\alpha-\beta-\lambda & \alpha & & \alpha \\
\alpha & \alpha & \ddots & & \alpha \\
\vdots & & & \ddots & \vdots \\
\alpha & \cdots & \cdots & \cdots & 1+\alpha-\beta-\lambda
\end{array}\right|
$$

By using a linear transformation on the rows and developing $P_{m}$ according to the first row, we obtain :

Journal home page: www.jafristat.net 


$$
\begin{aligned}
P_{m} & =\left|\begin{array}{ccccc}
1-\beta-\lambda-(1-\beta-\lambda) & 0 & \cdots & 0 \\
\alpha & 1+\alpha-\beta-\lambda & \alpha & & \alpha \\
\alpha & \alpha & \ddots & & \alpha \\
\vdots & & & \ddots & \vdots \\
\alpha & \cdots & \cdots & \cdots & 1+\alpha-\beta-\lambda
\end{array}\right| \\
& =(1-\beta-\lambda) P_{m-1}+\alpha(1-\beta-\lambda)^{m-1} Q_{m-1} .
\end{aligned}
$$

Let us define $P_{m}$ by induction. After a linear transformation on the rows, the second determinant $Q_{m-1}$ on the right-hand side becomes:

$$
Q_{m-1}=\left|\begin{array}{ccccc}
0 & -(1-\beta-\lambda) & 0 & \ldots & 0 \\
\alpha & 1+\alpha-\beta-\lambda & \alpha & & \alpha \\
\alpha & \alpha & \ddots & & \alpha \\
\vdots & & & \ddots & \vdots \\
\alpha & \ldots & \ldots & \ldots & 1+\alpha-\beta-\lambda
\end{array}\right|
$$

which implies that

$$
Q_{m-1}=(1-\beta-\lambda) Q_{m-2} \text { and } P_{m}=(1-\beta-\lambda) P_{m-1}+(1-\beta-\lambda) Q_{m-1}
$$

Hence

$$
Q_{m-1}=(1-\beta-\lambda)^{m-3} Q_{2} \text { where } Q_{2}=\left|\begin{array}{cc}
\alpha & \alpha \\
\alpha & 1+\alpha-\beta-\lambda
\end{array}\right|=\alpha(1-\beta-\lambda)
$$

so that

$$
Q_{m-1}=\alpha(1-\beta-\lambda)^{m-2}
$$

and

$$
P_{m}=(1-\beta-\lambda) P_{m-1}+\alpha(1-\beta-\lambda)^{m-1} .
$$

Then

$$
P_{m-1}=(1-\beta-\lambda) P_{m-2}+\alpha(1-\beta-\lambda)^{m-2} .
$$

Substitute this in (8) gives

$$
P_{m}=(1-\beta-\lambda)^{2} P_{m-2}+2 \alpha(1-\beta-\lambda)^{m-1} .
$$

We repeat this procedure for $P_{m-2}, P_{m-3} \ldots . . P_{3}$ to arrive at

$$
P_{m}=(1-\beta-\lambda)^{m-2} P_{2}+(m-2) \alpha(1-\beta-\lambda)^{m-1} .
$$


But

$$
P_{2}=(1-\beta-\lambda)(1+2 \alpha-\beta-\lambda)
$$

Then,

$$
P_{m}=(1-\beta-\lambda)^{m-1}(1+m \alpha-\beta-\lambda)
$$

We obtain in a similar way

$$
\operatorname{det}\left(Y-\lambda I_{m}+2 Z\right)=(1+2 \beta-\lambda)^{m-1}(1+m \alpha+2 \beta-\lambda) .
$$

Finally we have

$$
\mathbf{P}(\lambda)=(1-\beta-\lambda)^{2 m-2}(1+m \alpha-\beta-\lambda)^{2}(1+2 \beta-\lambda)^{m-1}(1+m \alpha+2 \beta-\lambda)
$$

By substituting $\alpha$ and $\beta$ with their real values, we obtain

$$
\begin{aligned}
\mathbf{P}(\lambda)= & \left(\frac{1}{m}-\lambda\right)^{2 m-2}\left(\frac{1}{m}+\frac{1}{m-1}-\lambda\right)^{2}\left(1+2 \frac{m-1}{m}-\lambda\right)^{m-1} \\
& \times\left(1+\frac{1}{m-1}+2 \frac{m-1}{m}-\lambda\right)
\end{aligned}
$$

We conclude that the smallest eigenvalue of $A$ is $s_{1}=\frac{1}{m}$ with achieves the proof.

Remark 2. In two colors case, the matrix $A$ is given by $A=\left[\begin{array}{cc}Y & Z \\ Z & Y\end{array}\right]$. Then

$$
\begin{aligned}
\operatorname{det}\left(\mathbf{A}-\lambda I_{2 m}\right)= & (1-\beta-\lambda)^{m-1}(1+m \alpha-\beta-\lambda) \\
& \times(1+\beta-\lambda)^{m-1}(1+m \alpha+\beta-\lambda)
\end{aligned}
$$

so that

$$
\begin{aligned}
\operatorname{det}\left(\mathbf{A}-\lambda I_{2 m}\right) & =\left(\frac{1}{m}-\lambda\right)^{m-1}\left(\frac{2 m-1}{m(m-1)}-\lambda\right)\left(\frac{2 m-1}{m}-\lambda\right)^{m-1} \\
& \times\left(\frac{2 m^{2}-2 m+1}{m(m-1)}-\lambda\right)
\end{aligned}
$$

and

$$
s_{1}=\frac{1}{m}
$$

Finally, we can easily see that the smallest eigenvalue of $A$ is $\frac{1}{|\Lambda|}$. This achieves the proof.

Acknowledgement: Thank for Pr. Azzouz Dermoune and the referee for helpful comment to this article.

Journal home page: www.jafristat.net 
A. Bey Touati, H. Zeghdoudi and H. Boutabia, Journal Afrika Statistika, Vol. 6, 2011, pages 372-380. Computation of spectral gap for a colored disordered lattice gas.

\section{References}

[1] Boudou, A., S., Caputo, P., Dai Pra, P. and Posta, G., 2006. Spectral gap estimates for interacting particle systems via a Bochner-type identity. J. Funct. Anal, 232, 1, 222-258.

[2] Cancrini, N. and Martinelli, F., 2000. On the spectral gap of Kawasaki dynamics under a mixing condition revisited. Probabilistic techniques in equilibrium and nonequilibrium statistical physics. J. Math. Phys. 41, 3, 1391-1423.

[3] Caputo, P. 2004. Spectral gap inequalities in product spaces with conservation laws. Stochastic analysis on large scale interacting systems, 53-88, Adv.Stud. Pure Math., 39, Math. Soc. Japan, Tokyo.

[4] Dermoune, A. and Heinrich, P. 2004. A small step towards the hydrodynamic limit of a colored disordered lattice gas. C. R. Math. Acad. Sci, Paris 339 , 7, 507-511.

[5] Dermoune, A. and Heinrich, P., 2005. Equivalence of ensembles for colored particles in a disordered lattice gas. Markov Processes Relat. Fields, 11, 405-424.

[6] Dermoune, A. and Heinrich, P., 2008. Spectral gap inequality for a colored disordered lattice gas. Lecture Notes in Mathematics, Vol. 41. Seminaire de Probabilites XLI, X, .462.

[7] Faggionato, A. and Martinelli, F., 2003. Hydrodynamic limit of a disordered lattice gas. Probab. Theory Related Fields, 127, (4), 535-608.

[8] Kovacs, I., Silver, D. S., and Williams, S. G., 1999. Determinants of Commuting-Block Matrices. The American Mathematical Monthly, 106, (10), 950-952.

[9] Lu, S. L. and Yau, H., 1993. Spectral gap and logarithmic Sobolev inequality for Kawasaki and Glauber dynamics. Comm. Math. Phys., 156, no. 2, 399-433

[10] Zeghdoudi, H. and Boutabia, H., 2009. Computation for the Canonical Measures of a Colored Disordered Lattice Gas and Spectral Gap. Journal of Mathematical Physics, 50,103302, 1-8. 